\title{
A Porthole over AKI: Cell Dynamics in Damage and Repair
}

\author{
Luca Bordoni Donato Sardella Ina Maria Schiessl \\ Department of Biomedicine, Aarhus University, Aarhus, Denmark
}

\section{Keywords}

Acute kidney injury · Chronic kidney disease · Renal regeneration · Fibrosis · Intravital imaging

\begin{abstract}
Acute kidney injury (AKI) is associated with an increased risk of CKD. Injury-induced multifaceted renal cell-to-cell crosstalk can either lead to successful self-repair or chronic fibrosis and inflammation. In this mini-review, we will discuss critical renal cell types acting as victims or executioners in AKI pathology and introduce intravital imaging as a powerful technique to further dissect these cell-to-cell interactions.
\end{abstract}

๑) 2020 S. Karger AG, Basel

\section{Introduction}

Acute kidney injury (AKI), generically classified by the loss of renal filtration capacity over $48 \mathrm{~h}$, affects $15 \%$ of hospitalized patients [1]. In most cases, the kidney selfrepairs to full recovery, while in others, incomplete repair and tissue deterioration impose the development of CKD [1]. The path chosen depends on the severity of the underlying etiology, which involves complex crosstalk between renal endothelial, epithelial, inflammatory, and interstitial cells, controlling damage and repair altogether [2]. Dissecting the nature and time dynamics of these cel-

karger@karger.com

(c) 2020 S. Karger AG, Basel

www.karger.com/nef

Karger! lular interactions is crucial to identify and inhibit pathways of AKI-to-CKD transition. Intravital imaging of the kidney is a powerful technique to study structural and functional alterations simultaneously at subcellular resolution $[3,4]$. Here, we introduce the major yet doublefaced AKI-contributing cell's dynamics and underline the significance of intravital imaging as a unique tool to address them in vivo.

\section{Necroinflammation}

Inflammation and tubulointerstitial infiltration of activated immune cells are a hallmark of early AKI. While important for removal of bacterial and necrotic debris from the renal milieu, excessive release of pro-inflammatory cytokines induces cellular damage, incomplete tubular repair, and fibrosis [5]. Understanding dynamic immune cell trafficking requires active blood perfusion. Intravital imaging allows several labeling techniques to investigate immune cells in the intact renal environment [3], enabling the tracking of critical endothelial-immune cell interactions, such as patrolling, rolling, and adhesion in real time (Fig. 1).

Contribution from the AKI and CRRT 2020 Symposium at the 25th International Conference on Advances in Critical Care Nephrology, Manchester Grand Hyatt, San Diego, CA, USA, February 24-27th, 2020. This symposium was supported in part by the NIDDK funded University of Alabama at Birmingham-University of California San Diego O'Brien Center for Acute Kidney Injury Research (P30DK079337). 


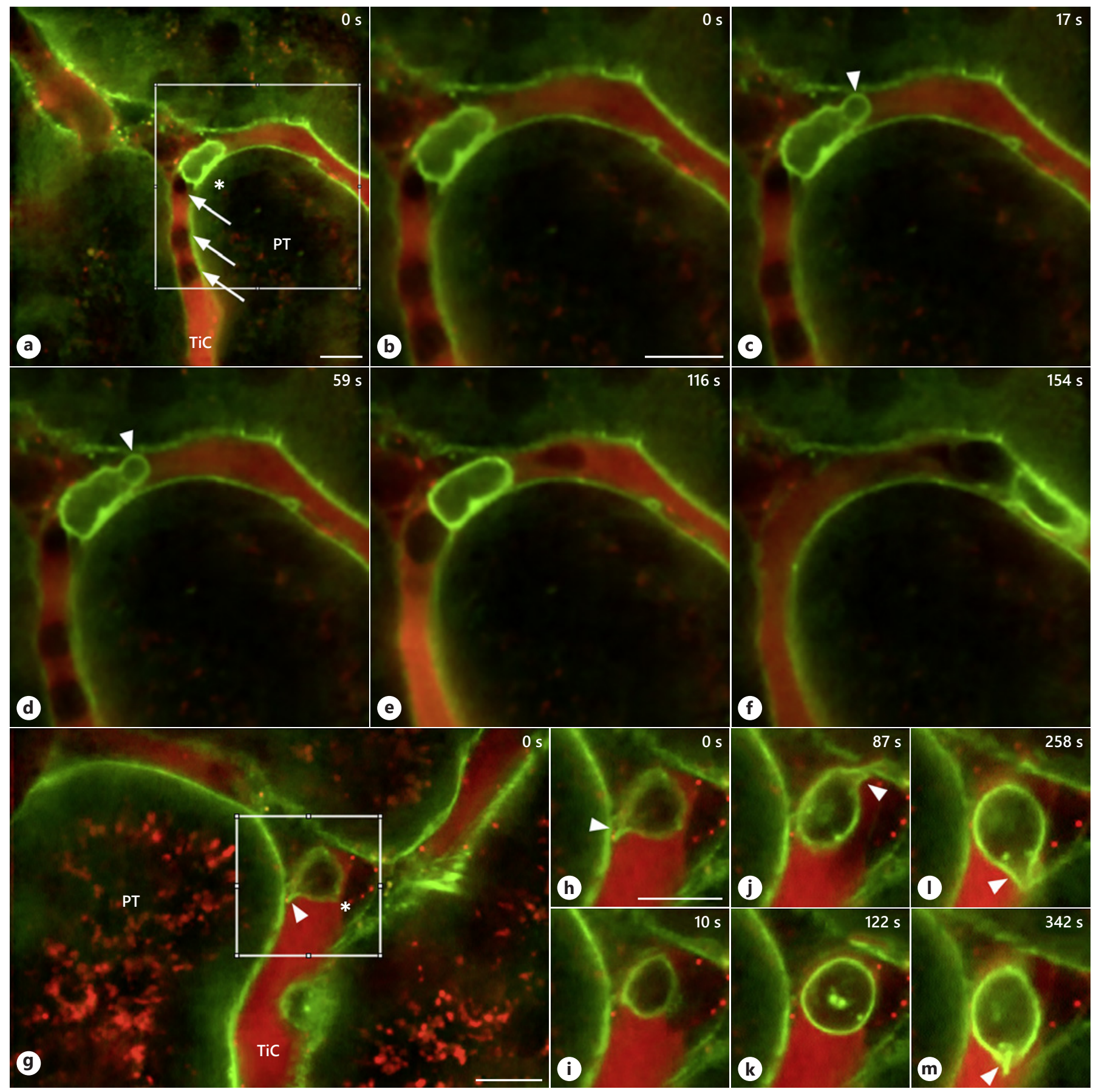

Fig. 1. Spinning disc confocal imaging of immune cell dynamics in a mouse kidney, showing leucocyte adhesion $\left({ }^{*}\right)$ with consequent erythrocyte halting (white arrows) (a-d) and leucocyte probing through podosome-like extensions (arrowheads, c, d, $\mathbf{g - m}$ ) in 2 different TiC, respectively $(\mathbf{a}-\mathbf{f}, \mathbf{g}-\mathbf{m})$, over indicated time. TiC

plasma and PT endocytic vesicles are labeled red using Alexa594conjugated albumin. TiC glycocalyx and leucocyte cell membranes are stained green using Alexa488-conjugated wheat germ agglutinin. $\mathrm{Bar}=10 \mu \mathrm{m}$ in all panels. PT, proximal tubule; TiC, tubulointerstitial capillaries. 

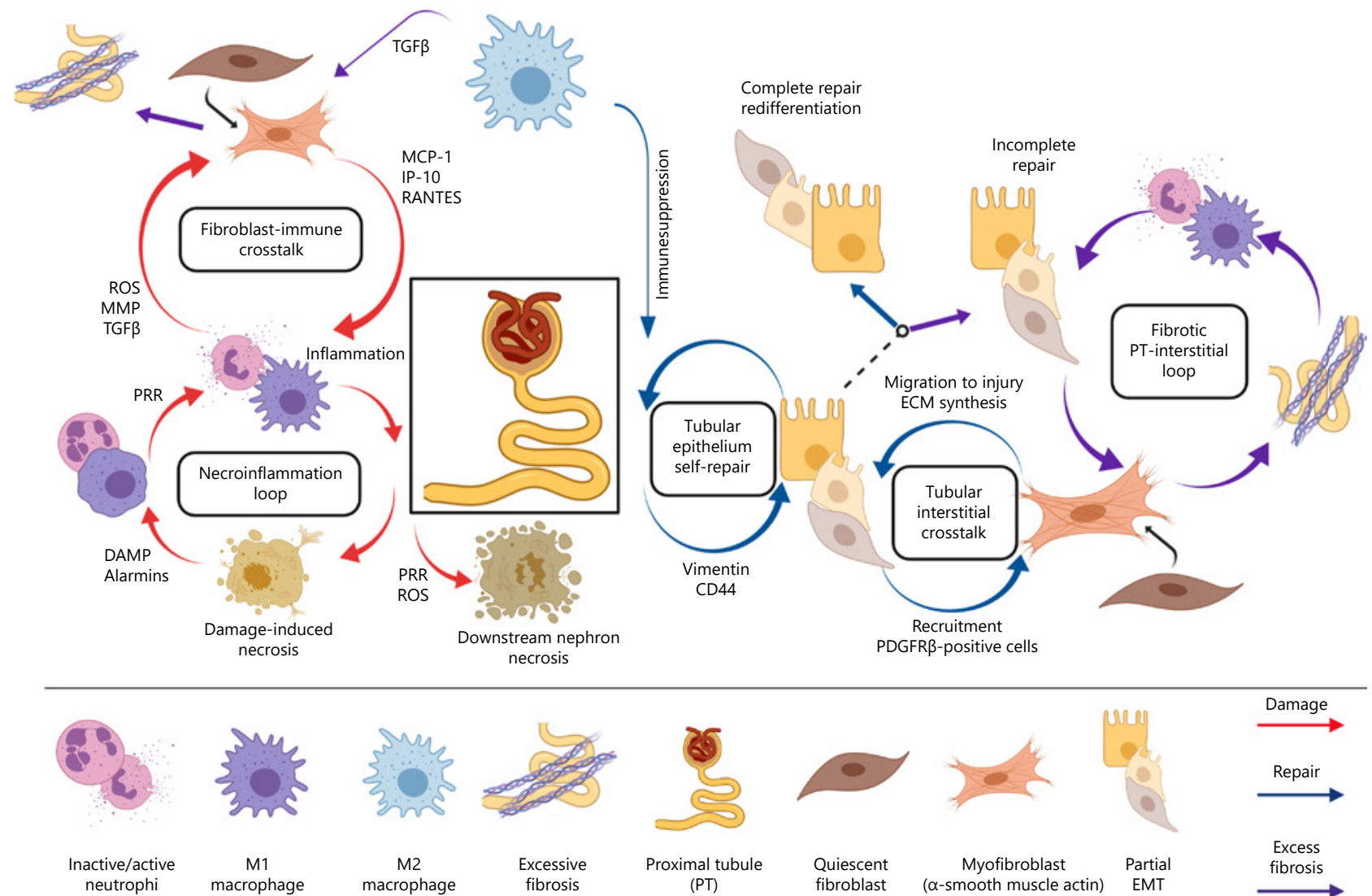
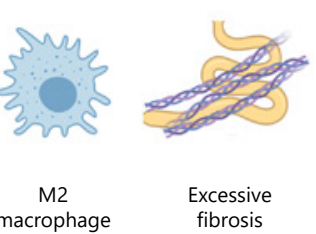

fibrosis

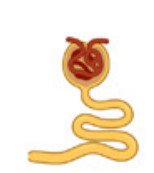

Proximal tubule

(PT)

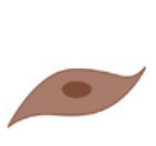

Quiescent

fibroblast

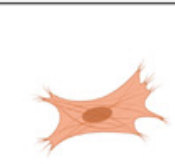

Myofibroblast $(\alpha$-smooth muscle actin)
Damage

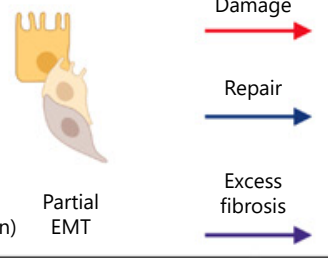

ROS: reactive oxygen species TGF $\beta$ : transforming growth factor beta MCP-1: monocyte chemotactic protein MMP: matrix metalloproteinases RANTES: CCL5 (chemokine ligand 5) PDGFR $\beta$ : platelet-derived growth factor-receptor $\beta$

Fig. 2. Crosstalk mechanisms described in this review (created with BioRender).

Proximal tubules (PTs) are the main injury site in AKI and crucial determinants of CKD progression [2]. However, PTs are also active protagonists of AKI pathology, amplifying inflammatory damage and regulated necrosis [6] as well as pathways of cellular repair [7]. Necrotic tubule cells contribute to immune cell activation through the release of cytosolic content, which, once displaced extracellularly, act as "danger-associated molecular patterns" (DAMPs), activating pattern recognizing receptors on leukocytes and monocytes $[5,6]$. Thus, PTs engage in immune cell chemoattraction and transendothelial migration.

In return, pattern recognizing receptor stimulation on PTs increases the synthesis and release of necrotic death mediators, perpetuating damage along the nephron [6].
Recent evidence showed that mechanisms of necrotic cell death are not merely passive as previously considered but tightly regulated through different molecular pathways, such as necroptosis and ferroptosis [8]. Thus, regulated PT necrotic cell death and subsequent DAMP release drive an inflammatory autoamplification loop between epithelial and immune cells (necroinflammation), which has been recognized as a key factor determining AKI pathology $[6,8]$. Intravital imaging is a powerful tool to study PT metabolic dysfunction, cell death, and immune cell interactions [4].

\section{Macrophages: Contribution to Damage and Repair}

Macrophages adopt flexible phenotypes during AKI as they transition from inflammatory cells (M1) to immu- 
nosupressors (M2) [9]. In early AKI, M1 differentiation occurs upon detection of immunogenic signals or DAMP molecules, leading to M1 accumulation in glomeruli and tubulointerstitium that evokes further recruitment of neutrophils and activated cytotoxic T cells [9]. In contrast, M2 macrophages contribute to immunosuppression, damage control, and tissue repair. Of note, M1/M2 classification likely oversimplifies a continuum of intermediate macrophage subphenotypes during AKI [9]. Thus, a distinct subpopulation of resident renal macrophages are also involved in kidney repair and angiogenesis [9], and in the zebra fish brain, a novel macrophagemediated mechanism of endothelial repair was recently identified using intravital imaging [10].

\section{Fibroblasts between Damage, Repair, and Fibrosis}

Fibroblasts are crucial protagonists of AKI. Immune cell stimulation promotes fibroblast differentiation into myofibroblasts, the main extracellular matrix (ECM)producing cells [11]. In return, activated fibroblasts sustain tissue inflammation by enhancing innate immune cell chemotaxis, promoting the expression of adhesion molecules in the endothelium, and retaining activated $\mathrm{T}$ cells in the tubulointerstitium [11]. This loop may result in elevated collagen production, and since excessive ECM deposition in the glomerular and tubulointerstitial compartments correlates with declined kidney function, expanding fibrosis has been considered a causal mechanism of CKD transition [7, 12].

However, anti-fibrotic drugs alone failed to ameliorate progressive kidney deterioration [13], and there is emerging evidence that fibrosis may accumulate as a byproduct of a tubular repair-enhancing tubulointerstitial crosstalk [7, 14, 15]. Rather than evolving in uncontrolled tubulointerstitial expansion, myofibroblast-derived fibrosis in AKI is restricted to areas of tubular repair [14]. Furthermore, a recent study using intravital two-photon microscopy showed the targeted migration of renal platelet-derived growth factor-receptor $\beta$-positive interstitial cells to the site of tubular injury and their wrapping around remodeling tubules [15]. Moreover, platelet-derived growth factor-receptor $\beta$ cells withdrew from the injury site upon tubular repair, and inhibition of their recruitment impaired tubular recovery [15]. This suggests a supporting role of fibrotic events during AKI, hypothesizing that tissue fibrosis during AKI-to-CKD transition results from a continually maintained positive loop of initially pro-regenerative epithelial-interstitial communication during incomplete repair $[7,14]$.

AKI: Cell Dynamics in Damage and Repair

\section{PTs' Incomplete Repair Triggers Fibrosis}

Tubular cell regeneration is associated with distinct morphological (flattening of cell shape) and functional (loss of albumin reuptake capacity) de-differentiation of resident tubular cells $[12,15]$. Transcriptionally, these cells undergo partial epithelial-to-mesenchymal transition through downregulation of epithelial (cadherin and megalin) and upregulation of mesenchymal (CD44 and vimentin) markers. This is associated with tubule cell migration, proliferation, and tissue repair $[7,12]$, as recently shown via serial intravital imaging of transgenic tubule-specific Confetti mouse kidneys [15]. In these mice, individual tubule cells express different fluorescent proteins, allowing their morphological and functional tracking over time [4]. Upon complete repair, tubule cells redifferentiate via downregulation of epithelial-to-mesenchymal transition markers $[12,15]$.

Dedifferentiated PTs stimulate myofibroblast activation, which in turn supports tubular wound healing [15]. However, overexposure to this crosstalk may promote inflammation and exacerbate tissue damage $[7,12]$. While temporary ECM deposition provides a useful 3D scaffold for tubular repair [14], its excessive production during incomplete repair may impact CKD transition [7]. Eventually, temporal and spatial dynamics of epithelial-interstitial crosstalk shift the balance toward AKI recovery or CKD (Fig. 2).

\section{Conclusions}

The outcome of AKI is determined by complex and bidirectional intercellular crosstalk. Hence, it is not surprising that unidirectional anti-inflammatory [11] or anti-fibrotic therapies [13] showed low efficacy. If not opposed at an early stage after AKI, necroinflammation and failure of tubular repair can lead to CKD. Intravital imaging of the post-AKI kidney is a unique tool to address the temporal and spatial dynamics of cellular events that direct the kidney toward recovery or $\mathrm{CKD}$.

\section{Disclosure Statement}

I.M.S. has received a grant from the Novo Nordisk Foundation. 


\section{References}

1 Bellomo R, Kellum JA, Ronco C. Acute kidney injury. Lancet. 2012 Aug 25;380(9843): 756-66.

2 Takaori K, Nakamura J, Yamamoto S, Nakata H, Sato Y, Takase M, et al. Severity and frequency of proximal tubule injury determines renal prognosis. J Am Soc Nephrol. 2016 Aug; 27(8):2393-406.

3 Hall AM, Molitoris BA. Dynamic multiphoton microscopy: focusing light on acute kidney injury. Physiology. 2014 Sep;29(5):33442.

4 Schiessl IM, Fremter K, Burford JL, Castrop $\mathrm{H}$, Peti-Peterdi J. Correction to: long-term cell fate tracking of individual renal cells using serial intravital microscopy. Methods $\mathrm{Mol}$ Biol. 2019 May 14.

5 Rabb H, Griffin MD, McKay DB, Swaminathan S, Pickkers P, Rosner MH, et al. Inflammation in AKI: current understanding, key questions, and knowledge gaps. J Am Soc Nephrol. 2016 Feb;27(2):371-9.
6 Mulay SR, Linkermann A, Anders HJ. Necroinflammation in kidney disease. J Am Soc Nephrol. 2016 Jan;27(1):27-39.

7 Schiessl IM. The role of tubule-interstitial crosstalk in renal injury and recovery. Semin Nephrol. 2020 Mar;40(2):216-31.

8 Sarhan M, von Mässenhausen A, Hugo C, Oberbauer R, Linkermann A. Immunological consequences of kidney cell death. Cell Death Dis. 2018 Jan 25;9(2):114.

9 Tang PM, Nikolic-Paterson DJ, Lan HY. Macrophages: versatile players in renal inflammation and fibrosis. Nat Rev Nephrol. 2019 Mar; 15(3):144-58

10 Liu C, Wu C, Yang Q, Gao J, Li L, Yang D, et al. Macrophages mediate the repair of brain vascular rupture through direct physical adhesion and mechanical traction. Immunity. 2016 May 17;44(5):1162-76.
11 Van Linthout S, Miteva K, Tschöpe C. Crosstalk between fibroblasts and inflammatory cells. Cardiovasc Res. 2014 May 1;102(2):25869.

12 E Oh, Humphreys BD. Fibrotic changes mediating acute kidney injury to chronic kidney diseasetransition. Nephron.2017;137(4):2647.

13 Allinovi M, De Chiara L, Angelotti ML, Becherucci F, Romagnani P. Anti-fibrotic treatments: a review of clinical evidence. $\mathrm{Ma}$ trix Biol. 2018 Aug;68-69:333-54

14 Kaissling B, Lehir M, Kriz W. Renal epithelial injury and fibrosis. Biochim Biophys Acta. 2013 Jul;1832(7):931-9.

15 Schiessl IM, Grill A, Fremter K, Steppan D, Hellmuth MK, Castrop H. Renal interstitial platelet-derived growth factor receptor- $\beta$ cells support proximal tubular regeneration. J Am Soc Nephrol. 2018 May;29(5):1383-96. 